PROCEEDINGS OF THE AMERICAN MATHEMATICAL SOCIETY

Volume 125, Number 3, March 1997, Pages 685-688

S 0002-9939(97)03612-5

\title{
A NEW CHARACTERIZATION OF SEMISIMPLE LIE ALGEBRAS
}

\author{
SAID BENAYADI
}

(Communicated by Roe Goodman)

\begin{abstract}
Using Casimir elements, we characterize the semisimple Lie algebras among the quadratic Lie algebras. This characterization gives, in particular, a generalization of a consequence of Cartan's second criterion.
\end{abstract}

\section{INTRODUCTION}

In this work we consider Lie algebras of finite dimension over a commutative algebraically closed field $\mathbb{K}$ of characteristic 0 .

Let $\mathfrak{g}$ be a Lie algebra and let $\mathfrak{z}(\mathfrak{g}), \mathfrak{R}(\mathfrak{g}), \mathfrak{U}(\mathfrak{g})$ denote the center of $\mathfrak{g}$, the radical of $\mathfrak{g}$, the universal enveloping algebra for $\mathfrak{g}$ respectively. A Lie algebra $\mathfrak{g}$ is called perfect if $\mathfrak{g}=[\mathfrak{g}, \mathfrak{g}]$. Letting $B$ be a bilinear form on $\mathfrak{g}$, we shall say that $B$ is an invariant scalar product on $\mathfrak{g}$ if it is symmetric, nondegenerate and invariant (i.e. $B([x, y], z)=-B(y,[x, z]))$. We shall say that a Lie algebra $\mathfrak{g}$ is quadratic if there exists an invariant scalar product on $\mathfrak{g}$.

Let $\mathfrak{g}$ be a quadratic Lie algebra and $B$ be an invariant scalar product on $\mathfrak{g}$. We choose bases $\left\{e_{i}\right\},\left\{f_{j}\right\}$ in the Lie algebra $\mathfrak{g}$ such that $B\left(e_{i}, f_{j}\right)=\delta_{i j}$ where $\delta_{i j}$ is the Kronecker symbol. Let $C_{B}$ be the element of $\mathfrak{U}(\mathfrak{g})$ defined by $C_{B}=\sum e_{i} f_{i}$. The element $C_{B}$ is called a Casimir element corresponding to the form $B$. By [Bo], the element $C_{B}$ is in the center of $\mathfrak{U}(\mathfrak{g})$ and is independent of the choice of bases $\left\{e_{i}\right\},\left\{f_{j}\right\}$. By [Bo], the adjoint representation of $\mathfrak{g}$ has a unique extension to a homomorphism $T$ of the associative algebra $\mathfrak{U}(\mathfrak{g})$ into the associative algebra $\operatorname{End}(\mathfrak{g})$. We shall say that $C_{B}$ is invertible (resp. nilpotent) if the linear map $T\left(C_{B}\right)$ is invertible (resp. nilpotent).

The fact that the Killing form of semisimple Lie algebra $\mathfrak{g}$ is an invariant scalar product on $\mathfrak{g}$ implies that $\mathfrak{g}$ is quadratic. But the class of quadratic Lie algebras contains strictly the class of semisimple Lie algebras. In [B1] and [B2], we have given some characterizations of the semisimple Lie algebras among certain classes of quadratic Lie algebras. Here, we characterize the semisimple Lie algebras among the quadratic Lie algebras using the Casimir elements. Our main result is Theorem 2: Let $\mathfrak{g}$ be a quadratic Lie algebra and let $B$ be an invariant scalar product on $\mathfrak{g}$. Then, the following two assertions are equivalent:

i) $\mathfrak{g}$ is semisimple;

ii) The Casimir element corresponding to the form $B$ is invertible.

Received by the editors May 4, 1995 and, in revised form, September 21, 1995.

1991 Mathematics Subject Classification. Primary 17B05, 17B20.

Key words and phrases. Semisimple Lie algebras, quadratic Lie algebras, Casimir elements. 
The above theorem generalizes a consequence of Cartan's second criterion: "If the Killing form is nondegenerate, then $g$ is semisimple". Note that the nondegeneration of the Killing form already implies that the Casimir element is invertible, hence the above theorem indeed gives a generalization of Cartan's result.

\section{Casimir elements of QUAdratic Perfect Lie Algebras}

The main result of this section states that the Casimir elements of a nonsemisimple quadratic perfect Lie algebra are not invertible.

Lemma 1. Let $\mathfrak{g}$ be a simple Lie algebra, $K$ its Killing form, and $B$ an invariant scalar product of $\mathfrak{g}$. Then, there exists an element $\lambda$ of $\mathbb{K} \backslash\{0\}$ such that $B=\lambda K$ and $C_{B}=\lambda^{-1} C_{K}$.

Using this straightforward lemma, we get the following proposition.

Proposition 1. Let $\mathfrak{g}$ be a semisimple Lie algebra and $B$ an invariant scalar product on $\mathfrak{g}$. Then $C_{B}$ is invertible.

We now show that the Casimir elements of a non-semisimple quadratic perfect Lie algebra without any nonzero semisimple ideal are nilpotent. We need the following two lemmas.

Lemma 2. Let $\mathfrak{g}$ be a perfect Lie algebra without any nonzero semisimple ideal, and let $\mathfrak{R}$ be its radical. If $\mathfrak{g}$ is quadratic, then $\mathfrak{R}^{\perp}=\mathfrak{z}(\mathfrak{R})$.

Proof. Let $B$ be an invariant scalar product on $\mathfrak{g}, x$ an element of $\mathfrak{R}$ and $y$ an element of $\mathfrak{z}(\mathfrak{R})$. Then $x=\sum_{i=1}^{n}\left[z_{i}, r_{i}\right]$ where $z_{i}$ is an element of $\mathfrak{g}$ and $r_{i}$ is an element of $\mathfrak{R}$ (because $[\mathfrak{g}, \mathfrak{R}]=\mathfrak{R}$ ). Thus $B(x, y)=\sum_{i=1}^{n} B\left(z_{i},\left[r_{i}, y\right]\right)=0$, hence $\mathfrak{z}(\mathfrak{R}) \subset \mathfrak{R}^{\perp}$. Let $\mathfrak{s}$ be a Levi subalgebra of $\mathfrak{g}$. Since $\mathfrak{R}^{\perp}$ is an ideal of $\mathfrak{g}$, by [Bo], $\mathfrak{R}^{\perp}=\mathfrak{s} \cap \mathfrak{R}^{\perp} \oplus \mathfrak{R} \cap \mathfrak{R}^{\perp}$. Then $\mathfrak{s} \cap \mathfrak{R}^{\perp}$ is a Levi subalgebra of $\mathfrak{R}^{\perp}$ and $\mathfrak{s} \cap \mathfrak{R}^{\perp}$ is an ideal of $\mathfrak{s}$. Letting $x \in \mathfrak{g}, y \in \mathfrak{R}^{\perp}$ and $z \in \mathfrak{R}$, then $[x, z] \in \mathfrak{R}$ and we have

$$
B([y, z], x)=B(y,[x, z])=0 .
$$

Since $B$ is nondegenerate, then $[y, z]=0$. Thus $\left[\mathfrak{R}^{\perp}, \mathfrak{R}\right]=\{0\}$. It follows that $\left[\mathfrak{s} \cap \mathfrak{R}^{\perp}, \mathfrak{R}\right]=\{0\}$. Consequently, $\mathfrak{s} \cap \mathfrak{R}^{\perp}$ is a semisimple ideal of $\mathfrak{g}$. So, $\mathfrak{s} \cap \mathfrak{R}^{\perp}=$ $\{0\}$ because $\mathfrak{g}$ is a Lie algebra without any nonzero semisimple ideal. It follows that $\mathfrak{R}^{\perp} \subset \mathfrak{R}$. The fact that $\left[\mathfrak{R}^{\perp}, \mathfrak{R}\right]=\{0\}$ implies that $\mathfrak{R}^{\perp} \subset \mathfrak{z}(\mathfrak{R})$, hence the result.

Lemma 3. Let $\mathfrak{g}$ be a perfect Lie algebra without any nonzero semisimple ideal, let $\mathfrak{R}$ be its radical and let $\mathfrak{s}$ be a Levi subalgebra of $\mathfrak{g}$. Assume that $\mathfrak{g}$ is quadratic and let $B$ be an invariant scalar product on $\mathfrak{g}$. Then there exists a vector subspace $V$ of $\Re$ which satisfies:

i) $[\mathfrak{s}, V] \subset V$;

ii) $\left.B\right|_{V \times V}$ is non-degenerate;

iii) $\mathfrak{R}=\mathfrak{z}(\mathfrak{R}) \oplus V$;

iv) $B(\mathfrak{s}, V)=\{0\}$.

Proof. We shall show that $B_{\mid A \times A}$ is non-degenerate where $A=\mathfrak{s} \oplus \mathfrak{z}(\mathfrak{R})$. Let $x=y+z$ be an element of $A$ where $y$ is an element of $\mathfrak{s}$ and $z$ is an element of $\mathfrak{z}(\mathfrak{R})$ such that $B(x, A)=\{0\}$. Then $B\left(y, z^{\prime}\right)=0$ for every element $z^{\prime}$ of $\mathfrak{z}(\mathfrak{R})$. By Lemma 2, it follows that $y=0$. Thus $x=z$ and $B(z, \mathfrak{s})=\{0\}$, which implies that $x=0$. We conclude that $B_{\mid A \times A}$ is non-degenerate. Consequently, $\mathfrak{g}=A \oplus A^{\perp}$. 
Next we show that $A^{\perp} \subset \mathfrak{R}$ and $\left[\mathfrak{s}, A^{\perp}\right] \subset A^{\perp}$. Indeed, the fact that $\mathfrak{z}(\mathfrak{R}) \subset A$ implies by Lemma 2 that $A^{\perp} \subset \mathfrak{R}$. Now consider $z \in \mathfrak{s}, x \in A^{\perp}$ and $y \in A$. Then $B([z, x], y)=-B(x,[z, y])=0$, which implies that $[z, x]$ is an element of $A^{\perp}$. Thus $\left[\mathfrak{s}, A^{\perp}\right] \subset A^{\perp}$. Since $\mathfrak{s} \subset A$, then $B\left(\mathfrak{s}, A^{\perp}\right)=\{0\}$. The nondegeneracy of $B$ implies that $\mathfrak{g}=A \oplus A^{\perp}$ and, consequently, $\mathfrak{g}=\mathfrak{s} \oplus\left(\mathfrak{z}(\mathfrak{R}) \oplus A^{\perp}\right)$. It follows that $\mathfrak{R}=\mathfrak{z}(\mathfrak{R}) \oplus A^{\perp}$ because $\mathfrak{R} \subset \mathfrak{z}(\mathfrak{R}) \oplus A^{\perp}$ and $\mathfrak{g}=\mathfrak{s} \oplus \mathfrak{R}$. So, $V=A^{\perp}$ satisfies all the requirements.

Next we prove:

Theorem 1. Let $\mathfrak{g}$ be a perfect Lie algebra without any nonzero semisimple ideal, and let $B$ be an invariant scalar product on $\mathfrak{g}$. Then $C_{B}$ is nilpotent.

Proof. There exists a vector subspace $V$ of $\mathfrak{g}$ which satisfies i), ii), iii) and iv) of Lemma 3. Thus, $\mathfrak{g}=\mathfrak{s} \oplus V \oplus \mathfrak{z}(\mathfrak{R})$ where $\mathfrak{s}$ (resp. $\mathfrak{R}$ ) is a Levi component (resp. the radical) of $\mathfrak{g}$.

By Lemma $2, \mathfrak{z}(\mathfrak{R})=\mathfrak{R}^{\perp}$, which implies that the map $\phi: \mathfrak{z}(\mathfrak{R}) \rightarrow \mathfrak{s}^{*}$ (resp. $\left.\psi: \mathfrak{s} \rightarrow \mathfrak{z}(\mathfrak{R})^{*}\right)$ defined by $\phi(x)=B(x,).($ resp. $\psi(x)=B(x,)$.$) for all x \in \mathfrak{z}(\mathfrak{R})$ (resp. $\mathfrak{s})$ is an invertible linear map.

Let $\left\{e_{1}, \ldots, e_{n}\right\}$ (resp. $\left.\left\{f_{1}, \ldots, f_{n}\right\}\right)$ be a basis of $\mathfrak{s}($ resp. $\mathfrak{z}(\mathfrak{R}))$. Then there exists a basis $\left\{l_{1}, \ldots, l_{n}\right\}$ (resp. $\left.\left\{k_{1}, \ldots, k_{n}\right\}\right)$ of $\mathfrak{z}(\mathfrak{R})$ (resp. $\left.\mathfrak{s}\right)$ such that $B\left(e_{i}, l_{j}\right)=\delta_{i j}$ (resp. $B\left(f_{i}, k_{j}\right)=\delta_{i j}$ ) for all $i, j \in\{1, \ldots, n\}$.

Let $\left\{v_{1}, \ldots, v_{m}\right\}$ be a basis of $V$. By Lemma 3 there exists a basis $\left\{w_{1}, \ldots, w_{m}\right\}$ of $V$ such that $B\left(v_{i}, w_{j}\right)=\delta_{i j}$ for all $i, j \in\{1, \ldots, m\}$. Then

$$
\begin{gathered}
C_{B}=\sum_{i=1}^{n} e_{i} l_{i}+\sum_{i=1}^{m} v_{i} w_{i}+\sum_{i=1}^{n} f_{i} k_{i}, \text { and } \\
T\left(C_{B}\right)=\sum_{i=1}^{n} a d_{\mathfrak{g}} e_{i} a d_{\mathfrak{g}} l_{i}+\sum_{i=1}^{m} a d_{\mathfrak{g}} v_{i} a d_{\mathfrak{g}} w_{i}+\sum_{i=1}^{n} a d_{\mathfrak{g}} f_{i} a d_{\mathfrak{g}} k_{i} .
\end{gathered}
$$

We now verify the following equation: $\left(T\left(C_{B}\right)\right)^{p}=\left(\sum_{i=1}^{m} a d_{\mathfrak{g}} v_{i} a d_{\mathfrak{g}} w_{i}\right)^{p}$, for every integer $p \geq 2$. Let us set $E=\sum_{i=1}^{n} e_{i} l_{i}, F=\sum_{i=1}^{m} v_{i} w_{i}$, and $G=$ $\sum_{i=1}^{n} f_{i} k_{i}$. Letting $x$ be an element of $\mathfrak{g}$, then $T\left(C_{B}\right)(x) \in \mathfrak{R}$ and it follows that $E\left(T\left(C_{B}\right)(x)\right)=G\left(T\left(C_{B}\right)(x)\right)=0$. Since $\left\{f_{1}, \ldots, f_{n}\right\}$ and $\left\{l_{1}, \ldots, l_{n}\right\}$ are bases of $\mathfrak{z}(\mathfrak{R})$, then $F\left(T\left(C_{B}\right)(x)\right)=F^{2}(x)$. Therefore, $\left(T\left(C_{B}\right)\right)^{2}(x)=F^{2}(x)$.

We assume that the equation is true for an integer $p \geq 2$, so that we have:

$$
\left(T\left(C_{B}\right)\right)^{(p+1)}(x)=E\left(F^{p}(x)\right)+F^{(p+1)}(x)+G\left(F^{p}(x)\right)
$$

Since $F^{p}(x) \in \Re$, it follows that $E\left(F^{p}(x)\right)=G\left(F^{p}(x)\right)=0$, consequently

$$
\left(T\left(C_{B}\right)\right)^{(p+1)}(x)=F^{(p+1)}(x) \text {. }
$$

Hence, the equation holds for every $p \geq 2$ by induction.

Since $\mathfrak{g}$ is perfect, it follows that $\mathfrak{R}$ is nilpotent. By the theorem of Lie (see [Bo]), there exists a basis $D$ of $\mathfrak{g}$ with respect to which the matrix of $a d_{\mathfrak{g}} x$ is triangular with all the elements on the diagonal equal to zero, for all $x$ in $\mathfrak{R}$. Consequently, there exists an integer $p \geq 2$ such that $\left(\sum_{i=1}^{m} a d_{\mathfrak{g}} v_{i} a d_{\mathfrak{g}} w_{i}\right)^{p}=0$. We conclude that $C_{B}$ is nilpotent.

Corollary. Let $\mathfrak{g}$ be a perfect, quadratic Lie algebra, which is not semisimple, and let $B$ be an invariant scalar product of $\mathfrak{g}$. Then $C_{B}$ is not invertible. 
Proof. There exists an ideal $\mathfrak{I}$ of $\mathfrak{g}$ such that $\mathfrak{g}=\mathfrak{S} \oplus \mathfrak{I}$ where $\mathfrak{S}$ is a maximal semisimple ideal of $\mathfrak{g}$.

The fact that $B$ is an invariant scalar product on $\mathfrak{g}$ implies that $H=\left.B\right|_{\mathfrak{S} \times \mathfrak{S}}$ (resp. $L=\left.B\right|_{\mathfrak{I} \times \mathfrak{I})}$ ) is an invariant scalar product on $\mathfrak{S}($ resp. $\mathfrak{I})$, and $B(\mathfrak{S}, \mathfrak{I})=\{0\}$.

By Theorem 1, $C_{L}$ is nilpotent, and by Proposition $1, C_{H}$ is invertible. Let $T$ be the representation of $U(\mathfrak{g})$ in $\mathfrak{g}$. Then:

i) $\left.T\left(C_{H}\right)\right|_{\mathfrak{S}}$ is invertible and $T\left(C_{H}\right)(\mathfrak{I})=\{0\}$;

ii) $T\left(C_{L}\right)(\mathfrak{S})=\{0\}$ and $\left.T\left(C_{L}\right)\right|_{\mathfrak{I}}$ is nilpotent;

iii) $T\left(C_{H}\right) \circ T\left(C_{L}\right)=T\left(C_{L}\right) \circ T\left(C_{H}\right)=0$;

iv) $T\left(C_{B}\right)=T\left(C_{H}\right)+T\left(C_{L}\right)$.

Thus $T\left(C_{L}\right)$ is nilpotent, which implies that there exists an integer $p \geq 1$ such that $\left(T\left(C_{L}\right)\right)^{p}=0$ and $\left(T\left(C_{B}\right)\right)^{p}=\left(T\left(C_{H}\right)\right)^{p}$. Hence $\mathfrak{I}$ is contained in $\operatorname{Ker}\left(T\left(C_{B}\right)\right)^{p}$. Since $\mathfrak{g}$ is not semisimple, $\mathfrak{I}$ is nonzero, and therefore $\left(T\left(C_{B}\right)\right)^{p}$ is not invertible. Hence $T\left(C_{B}\right)$ is not invertible.

\section{A NECESSARY AND SUFFICIENT CONDITION OF SEMISIMPLICITY OF QUADRATIC LIE ALGEBRAS}

Now we are in position to state our main result:

Theorem 2. Let $\mathfrak{g}$ be a quadratic Lie algebra, B an invariant scalar product on $\mathfrak{g}$. Then $\mathfrak{g}$ is semisimple if and only if the Casimir element $C_{B}$ corresponding to the form $B$ is invertible.

Proof. (a) If $\mathfrak{g}$ is semisimple, then by Proposition $1, C_{B}$ is invertible.

(b) Suppose that $C_{B}$ is invertible; then $\mathfrak{z}(\mathfrak{g})=\{0\}$. It is easy to see that $[\mathfrak{g}, \mathfrak{g}]^{\perp}=$ $\mathfrak{z}(\mathfrak{g})$, and therefore $[\mathfrak{g}, \mathfrak{g}]=\mathfrak{g}$. By the corollary of Theorem $1, \mathfrak{g}$ is semisimple.

Remark. It is well known that the invertibility of the Casimir elements is very useful in the cohomological theory of semisimple Lie algebras. The above theorem shows that, in the case of quadratic Lie algebras which are not semisimple, other methods would be necessary for the cohomological theory.

\section{ACKNOWLEDGMENTS}

I would like to thank D. Arnal, B. Bekka, H. Benamor, C. Bennis, M. Bordemann, B. Cahen, M. Flato, P. Ntolo, G. Pinczon for very interesting discussions, and I. Shafrir for a careful reading of the manuscript. I thank also the referee for his remarks which improved the readability of this article.

\section{REFERENCES}

[B1] S. Benayadi, Une propriété nécessaire et suffisante pour qu'une algèbre de, C.R. Acad. Sci. Paris, t. 319 (1994), 1155-1158. MR 95m:17009

[B2] - Structures de certaines algèbres de Lie quadratiques, Comm. in Algebra (to appear). MR 96h:17004

[Bo] N. Bourbaki, Groupes et algèbres de Lie, Hermann, Paris, 1971. MR 42:6159

Universite de Metz, Département de Mathematiques, U.R.A. CNRS Nº 399, Ile du Saulcy, F-57045 Metz Cedex 01, France

E-mail address: benayadi@poncelet.univ-metz.fr 